\title{
Formation of a Metallic Contact: Jump to Contact Revisited
}

\author{
C. Untiedt, ${ }^{1, *}$ M. J. Caturla, ${ }^{1}$ M. R. Calvo, ${ }^{1}$ J. J. Palacios, ${ }^{1}$ R. C. Segers,${ }^{2}$ and J. M. van Ruitenbeek ${ }^{2}$ \\ ${ }^{1}$ Departamento de Física Aplicada, Universidad de Alicante, Campus de San Vicente del Raspeig, E-03690 Alicante, Spain \\ ${ }^{2}$ Kamerlingh Onnes Laboratorium, Leiden University, P.O. Box 9504, NL-2300 RA Leiden, The Netherlands
}

(Received 7 November 2006; published 15 May 2007)

\begin{abstract}
The transition from tunneling to metallic contact between two surfaces does not always involve a jump, but can be smooth. We have observed that the configuration and material composition of the electrodes before contact largely determine the presence or absence of a jump. Moreover, when jumps are found preferential values of conductance have been identified. Through a combination of experiments, molecular dynamics, and first-principles transport calculations these conductance values are identified with atomic contacts of either monomers, dimers, or double-bond contacts.
\end{abstract}

DOI: 10.1103/PhysRevLett.98.206801

Matter at the atomic scale has attracted much attention during the past two decades. Not only because of the new properties arising when size is decreased, but also because the mechanisms found there can help us understand behavior at the macroscopic scale. This is the case of atomic contacts where many new and unexpected phenomena have been found [1] and, at the same time, have provided clues about many technological problems in the macroscopic scale. A clear example would be the study of adhesion or friction which are consequences of the formation of a contact between two bodies, a process which always involves the formation of at least one atomic contact [2].

The formation of a contact between two metallic electrodes can be studied by measuring the electronic transport through them. It has been observed that right before the electrodes are brought into contact, electrons can tunnel from one electrode to the other. This tunneling current will increase exponentially while the electrodes come together and at some point a jump to contact (JC) occurs: the electrode separation becomes unstable and a sudden increase of the current takes place because of the formation of a single-atom contact $[1,3]$ with a conductance which is determined by the number of conducting channels through the atomic contact $n$ and the transmission of each of these $T_{n}$ in the form $G=G_{0} \sum T_{n}$ being $G_{0}$ the quantum unit of conductance $\left(G_{0}=2 e^{2} / h\right)$. When the electrodes are then separated, a hysteretic behavior is observed in which the contact breaks in a jump to form a vacuum gap while relieving the elastic tension accumulated [4].

The process described above has been seen as the general rule in the formation of a metallic contact with a few exceptions to be understood [1]. It has been reported that in some cases the jump does not occur and, instead, there is a continuous increase of current from tunneling to contact, after which it saturates, forming a plateau showing no hysteresis when the electrodes are separated. Examples of this behavior have accumulated and presently have been reported for the cases of a W(111)-Au(111) contact [5], W nanocontacts [6], and for the case when a STM tip approaches $\mathrm{Ag}$ and $\mathrm{Cu}$ adatoms on (111) surfaces [7].
PACS numbers: 73.63. $-\mathrm{b}, 62.25 .+\mathrm{g}, 68.35 . \mathrm{Np}, 68.65 .-\mathrm{k}$

Here, the formation of a metallic contact for various metals ( $\mathrm{Au}, \mathrm{Pt}, \mathrm{Ag}, \mathrm{Ir}, \mathrm{W}$, and Ni) is investigated. A statistical analysis using density plots has been developed that, combined with atomistic simulations and $a b$ initio calculations of the electronic transport, provide new insight into the problem. The absence of a JC is not an exception, but it occurs for many metals, in particular for $\mathrm{Ni}, \mathrm{W}$, and Ir.

In order to control the formation of the atomic contacts we have employed a homemade high stability scanning tunneling microscope (STM) and mechanically controlled break junctions (MCBJ) [1], both techniques giving the same results for the different materials. In STM experiments, the tip of the STM is contacted to a sample made of the same material. The MCBJ experiments start from a notched wire that is glued to a bending substrate which bends using a piezoelement. At some point the wire breaks at the notch forming two fresh surfaces which are brought back together to form the atomic contacts. All experiments were performed at low temperatures $(4.2 \mathrm{~K})$ and under cryogenic vacuum conditions. The traces of conductance were taken at constant bias voltage (typically at $10 \mathrm{mV}$ ), which has been shown [8] to have negligible effect on the JC. A current amplifier in the range of $\mathrm{mA}$ was used to measure the current between the electrodes while their distance was controlled using the piezoelement. Calibration of interelectrode displacements were done by means described elsewhere [9].

Typical traces are shown in Fig. 1. The conductance increases exponentially as the electrodes are brought together, followed by a plateau of conductance that reveals the formation of an atomic contact. In previous works $[1,3-8,10,11]$ this process has been studied experimentally, mainly by looking at individual traces of conductance. Although this provides some information on the process, it is not suitable for quantitative conclusions. Here we have used another approach: First, we take a conductance histogram from a few thousands of traces of conductance. The conductance histogram is used to identify the conductance at which the contact is formed $\left(G_{c}\right)$. 


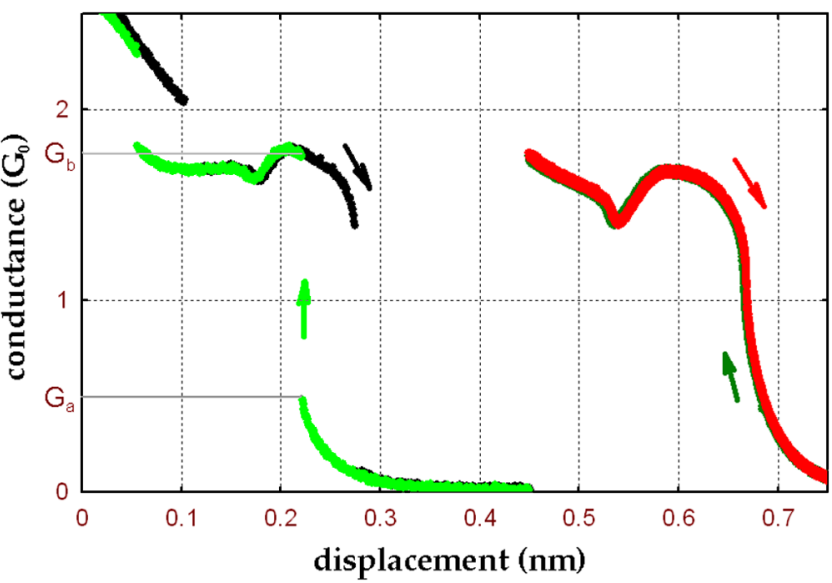

FIG. 1 (color online). Examples of traces of conductance obtained with a MCBJ for Ir atomic contacts as a function of the interelectrode displacement at $4.2 \mathrm{~K}$ showing a jump to contact (left) or a smooth transition (right). Arrows indicate the direction of the displacement of the electrodes. When there is a jump to contact, the jump starts from $G_{a}$ and ends at $G_{b}$. The curve on the right has been shifted horizontally for clarity.

Next, for each contact closing trace we automatically record two values, namely, the value of conductance below $G_{c}$ at which the steepest jump of conductance occurs $\left(G_{a}\right)$ and the value of conductance immediately following the jump $\left(G_{b}\right)$. Finally, these values are used to construct a density plot such as the one in the left-hand panel of Fig. 2. The density of pairs $\left(G_{a}, G_{b}\right)$ is plotted in color coding with $G_{a}$ and $G_{b}$ along the horizontal and vertical axis, respectively. The pairs $\left(G_{a}, G_{b}\right)$ for which a JC occurs with maximal probability are shown as a maximum in this density plot.

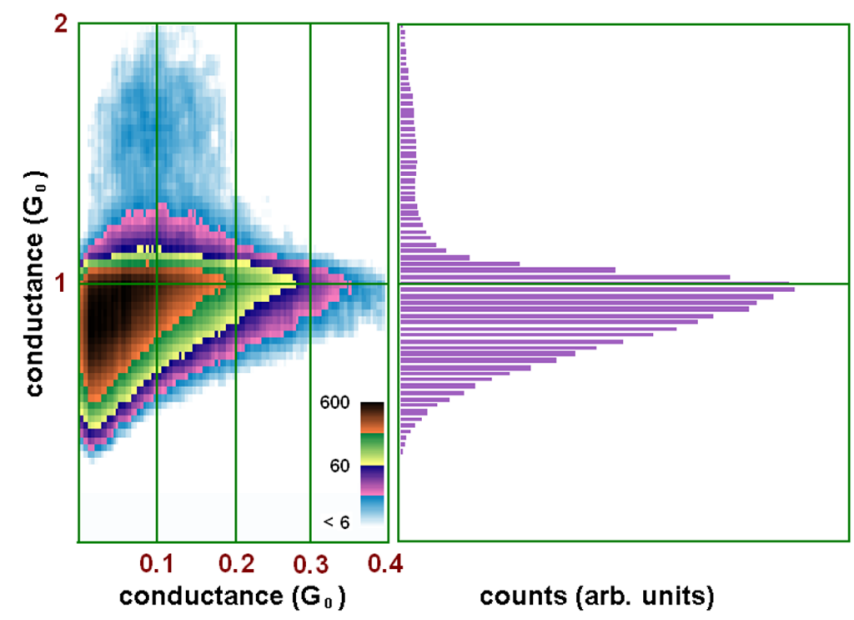

FIG. 2 (color online). Analysis of the steepest jump of conductance before the formation of a metallic contact for the case of gold, made from more than 300000 conductance traces. The left-hand panel shows a density plot in logarithmic scale, where the horizontal axis represents the conductance at which the jump takes place and the vertical axis shows the conductance of the contact formed. The right-hand panel shows the corresponding histogram of the conductance of the contact formed after the jump.
From all metals studied, three characteristic behaviors have been identified. Figure 2 shows our results for the case of $\mathrm{Au}$ from an analysis of more than 300000 traces. First we notice that there are no data near the line $G_{a}=G_{b}$, showing that there is always a JC. We observe a first maximum at the conductance $(0.03,0.87)$ in units of $G_{0}$, which means that in most of the cases the tunneling regime ends at a conductance of $0.03 G_{0}$ and then the conductance jumps to a value of $0.87 G_{0}$. The distribution for the jumps is quite broad for this maximum, especially in $G_{a}$. Further analysis shows that this peak is most likely the superposition of one at $(0.01,0.83)$ and another at $(0.05,0.94)$. We notice a second maximum at $(0.09,1.6)$, which corresponds to $7 \%$ of the data, as calculated from the distribution shown in the right-hand panel of Fig. 2.

In the case of $\mathrm{W}$ our observations coincide with those of Ref. [6] (made with the MCBJ technique); i.e., the JC is nearly always absent, as seen in the left-hand panel of Fig. 3. The evolution of the traces of conductance as the contact is stretched is completely different from those for Au since there is not a sharp transition between conductance plateaus. In the density plot we observe most of the data concentrated near the line defined by $G_{b}=G_{a}$. Although there are some abrupt transitions, these show no reproducibility and are seen in the density plot as the scarce data away from the $G_{b}=G_{a}$ line.

Finally, in the case of Ir, shown in the right-hand panel of Fig. 3 we find two maxima, one clearly shown at $(1.1,2.4) G_{0}$ and another on the $G_{a}=G_{b}$ line. The first maximum corresponds to a $\mathrm{JC}$ as observed for Au. The conductance at which the contact is formed is more than twice that of Au because of the higher valence of Ir with respect to $\mathrm{Au}$ [12]. The valence of the metal determines the number of electronic channels through the contact, and each channel contributes a conductance with a maximum of the quantum unit $G_{0}$. Note that the most probable

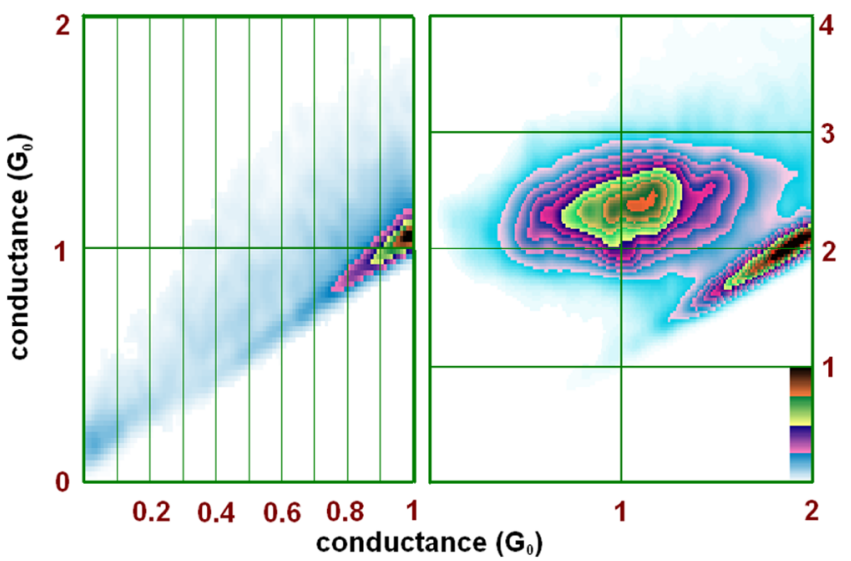

FIG. 3 (color online). Analysis of the steepest jump of conductance before the formation of a metallic contact for the case of W (left) and Ir (right) made from more than 10000 and 70000 conductance traces, respectively. The horizontal axis represents the conductance at which the jump takes place and the vertical axis shows the conductance of the contact formed. 
TABLE I. Summary of our results for the different measured materials. $G_{c}$ is the position of the first peak in the conductance histograms, JC gives the percentage of curves showing a jump to contact, and the last column shows the positions of the maxims in the density plots.

\begin{tabular}{ccrc}
\hline \hline Metal & $\begin{array}{c}G_{c} \\
\left.\text { (units of } G_{0}\right)\end{array}$ & JC & Maxima $\left(G_{a}, G_{b}\right)\left(\right.$ units of $\left.G_{0}\right)$ \\
\hline $\mathrm{Au}$ & 1.0 & $100 \%$ & $(0.01,0.87) ;(0.05,0.94) ;(0.09,1.6)$ \\
$\mathrm{Pt}$ & 1.5 & $100 \%$ & $(0.13,1.4) ;(0.19,2.0) ;(0.35,3.1)$ \\
$\mathrm{Ag}$ & 1.0 & $100 \%$ & $(0.07,1.0) ;(0.2,1.6)$ \\
$\mathrm{Ir}$ & 2.0 & $79 \%$ & $(1.1,2.4)$ \\
$\mathrm{Ni}$ & 1.3 & $75 \%$ & $(0.2,1.2)$ \\
$\mathrm{W}$ & 1.0 & $0 \%$ & \\
\hline \hline
\end{tabular}

conductance in the tunneling regime just before the Ir contact is formed is higher than one quantum of conductance. The second peak is located close to the line $G_{b}=$ $G_{a}$ and shows the $21 \%$ of cases when the JC is absent or is too small to be observed, which is similar to the behavior we observed for W.

These three examples are representative of what happens during contact formation. We have analyzed other materials and summarize our results in Table I.

Table I shows, for the different materials, the conductance of the first plateau, the percentage of cases having a $\mathrm{JC}$, and the positions of the maxima that can be clearly identified from the density plots. Note that for Pt three peaks are observed, like for $\mathrm{Au}$, while for $\mathrm{Ag}$ only two were resolved. The experiments were done using both techniques, STM and the MCBJ, except Ir and Pt (only MCBJ) and W (only STM).

The process of JC is related to the mechanical stiffness of the contact in competition with the attractive binding forces between the atoms to be contacted. Atomistic simulations $[2,13,14]$ have shown that when surfaces are brought into contact a jump occurs due to the adhesion forces between the metallic layers. However, this jump depends on the stiffness of the material and the geometry of the surfaces involved. For example, we have performed simple static calculations using embedded atom potentials for different surface geometries. The surfaces are shifted rigidly each step and then relaxed using conjugate gradient algorithms. From these calculations we observe a clear jump between two perfect (100) surfaces as well as for an atomically sharp tip approaching a perfect surface. However, the transition is smooth when two tips approach each other.

In the case of the nanocontacts described above, it is therefore expected that the existence of a $\mathrm{JC}$ will depend on the shape of the surfaces before contact. In order to understand this effect we have performed molecular dynamics simulations of the elongation of nanowires of $\mathrm{Au}$ and $\mathrm{W}$ until fracture. These fractured structures are then dynamically brought into contact. The simulations consisted of 525 and 437 atoms for $\mathrm{Au}$ and $\mathrm{W}$, respectively, where three atomic layers at the top and bottom of the simulation box were displaced every 1000 time steps by a fixed distance, resulting in a velocity of $1 \mathrm{~m} / \mathrm{s}$, characteristic of these types of calculations [15]. The simulation temperature was fixed to $4.2 \mathrm{~K}$. An embedded-atom method potential was used for the case of $\mathrm{Au}$ [16] while a Finnis-Sinclair potential was used for $\mathrm{W}$ [17]. A total of 25 cases were computed both for $\mathrm{Au}$ and $\mathrm{W}$. The atoms that first come into contact were identified considering a cutoff distance between first and second nearest neighbors. The distance between these atoms is then calculated as the two sides approach. Figure 4 shows the distance between the pairs of atoms that first come into contact, for two different configurations. Notice that in both cases there is a jump at contact. This occurs for all simulations performed for Au. The insets of Fig. 4 show the configurations at the point of contact for these two cases. In the left-hand panel the contact is made by two atoms, one on each side, forming a dimer, while in the right-hand panel a single atom (monomer) from one side contacts three atoms on the opposite side. From the 25 cases simulated $72 \%$ dimers are formed at contact, $20 \%$ monomers, while $8 \%$ are contacts through two parallel dimers or a double bond.

In order to verify whether or not the three configurations correspond to the three experimental peaks observed, we
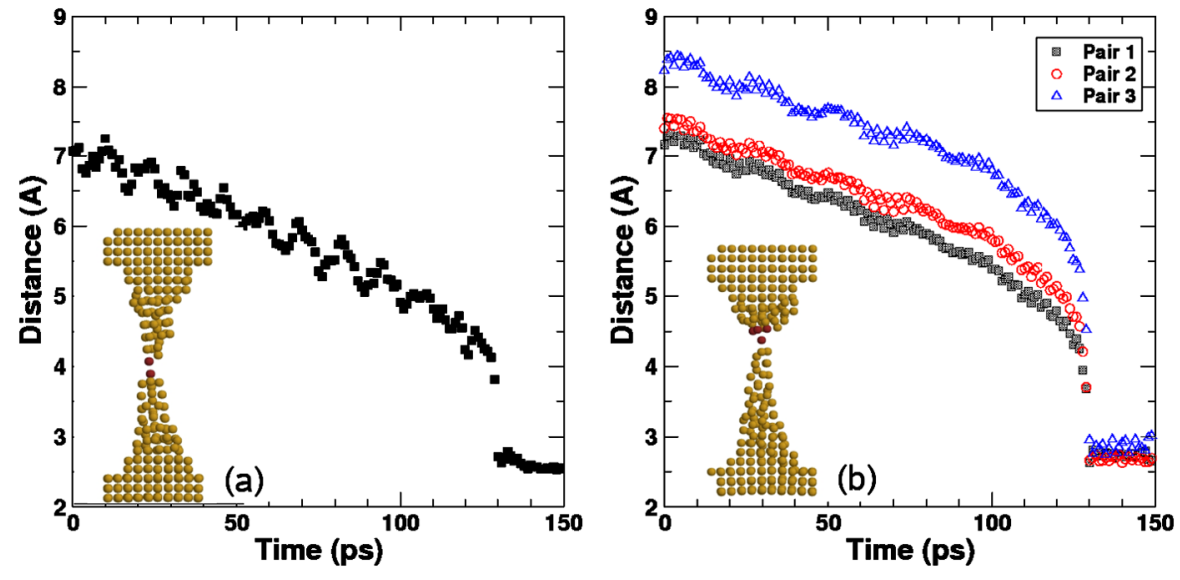

206801-3
FIG. 4 (color online). Distances between the pairs of atoms that first come into contact during the approach for two different configurations for $\mathrm{Au}$ obtained from molecular dynamics simulations. The most common configuration (a) consists of a dimer, while (b) shows the case of a monomer. The insets show the positions of the atoms. Dark atoms are those at contact. 


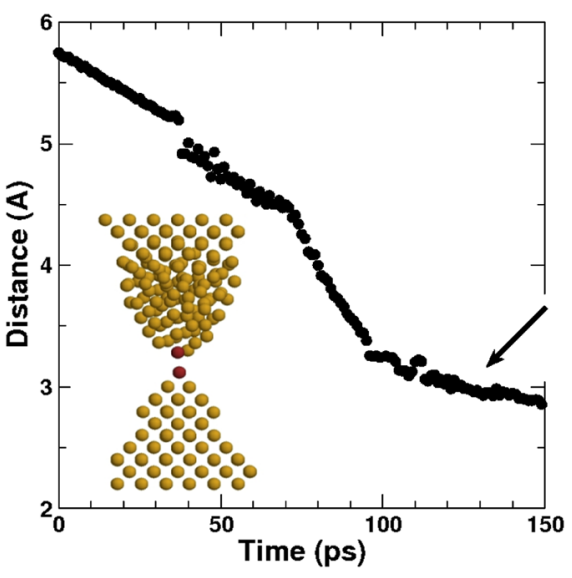

FIG. 5 (color online). Distances between the two atoms that first come into contact for $\mathrm{W}$, as obtained from a molecular dynamics simulation. The inset shows the positions of the atoms. Dark spheres are those atoms at contact. Contact (defined with the cutoff between first and second nearest neighbors) occurs at 130 ps. Notice the different scale with respect to Fig. 4.

have carried out first-principles quantum transport calculations for the conductance using the transport code ALACANT [18] for representative cases in all three configurations mentioned above. We have used either local density or generalized gradient corrected approximations for the exchange-correlation potential and pseudopotential basis sets with system sizes of up to 100 atoms. Although a statistical analysis is not feasible, the results confirm that dimers present a conductance upper limit of one quantum of conductance which lie within the experimental distribution. For the case of the monomer and double-bond contacts our results confirm higher values of conductance, again within the experimental distribution.

Finally, W was studied by molecular dynamics in order to understand those cases where no JC occurs. Figure 5 shows a particular configuration for $\mathrm{W}$ at the point of contact. Unlike $\mathrm{Au}$, for $\mathrm{W}$ there are configurations for which a jump is not observed at contact. These configurations are closer to two ideal tips than those found for Au. For a brittle material such as $\mathrm{W}$ much sharper and ordered structures are expected than for more ductile materials such as Au. In fact, as pointed out previously [6], the elastic properties of these metals play an important role in the presence of a JC.

In conclusion, a systematic analysis of the occurrence of a jump to contact in different metals has been performed using MCBJ and STM experiments, both techniques giving similar results. These experiments demonstrate the absence of a jump to contact for several metals. The results are interpreted using atomistic calculations of $\mathrm{Au}$ and $\mathrm{W}$. The geometry of the electrodes before contact seems to play a very significant role in determining whether a JC occurs, and this shape is related to the elastic properties of the metals. Consequently, softer metals such as Au or Ag always present a JC, while more brittle ones such as $\mathrm{W}$ do not. Moreover, with the help of first-principles transport calcu- lations we have been able to correlate the preferential values of conductance measured at JC in Au with three distinct configurations: a dimer, a monomer, and a double bond.

This work is part of the research program of the "Stichting FOM." Financial support through Grants No. FIS2004-02356 and No. MAT2005-07369-C03-1 of the Spanish MEC, and No. ACOMP06/138 of the Generalitat Valenciana is acknowledged. C.U. and M. J.C. acknowledge a Ramón y Cajal grant of the Spanish MCyT. We are grateful to David Jacob, S. Vieira and his group, and J. L. McDonald for technical, scientific, and computer support.

*Electronic address: untiedt@ua.es

[1] N. Agraï, A. Levy-Yeyati, and J. M. van Ruitenbeek, Phys. Rep. 377, 81 (2003).

[2] U. Landman, W. D. Luedtke, N.A. Burnham, and R. Colton, Science 248, 454 (1990).

[3] J. K. Gimzewski and R. Möller, Phys. Rev. B 36, 1284 (1987).

[4] G. Rubio, N. Agraït, and S. Vieira, Phys. Rev. Lett. 76, 2302 (1996).

[5] G. Cross, A. Schirmeisen, A. Stalder, P. Grutter, M. Tschudy, and U. Durig, Phys. Rev. Lett. 80, 4685 (1998).

[6] A. Halbritter, Sz. Csonka, G. Mihály, E. Jurdik, O. Yu. Kolesynchenko, O. I. Shklyarevskii, S. Speller, and H. van Kempen, Phys. Rev. B 68, 035417 (2003).

[7] L. Limot, J. Kröger, R. Berndt, A. García-Lekue, and W. A. Hofer, Phys. Rev. Lett. 94, 126102 (2005).

[8] C. Sirvent, J. G. Rodrigo, N. Agraït, and S. Vieira, Physica (Amsterdam) 218B, 238 (1996).

[9] C. Untiedt, A. I. Yanson, R. Grande, G. Rubio-Bollinger, N. Agraï, S. Vieira, and J. M. van Ruitenbeek, Phys. Rev. B 66, 085418 (2002).

[10] C. Sirvent, J. G. Rodrigo, S. Vieira, L. Jurczyszyn, N. Mingo, and F. Flores, Phys. Rev. B 53, 16086 (1996).

[11] V. Rodrigues, T. Fuhrer, and D. Ugarte, Phys. Rev. Lett. 85, 4124 (2000).

[12] E. Scheer, N. Agraït, J.C. Cuevas, A. Levy-Yeyati, B. Ludoph, A. Martín-Rodero, G. Rubio-Bollinger, J. M. van Ruitenbeek, and C. Urbina, Nature (London) 394, 154 (1998).

[13] J. B. Pethica and A.P. Sutton, J. Vac. Sci. Technol. A 6, 2490 (1988).

[14] J. R. Smith, G. Bozzolo, A. Banerjea, and J. Ferrante, Phys. Rev. Lett. 63, 1269 (1989).

[15] M. R. Sorensen, M. Brandbyge, and K. W. Jacobsen, Phys. Rev. B 57, 3283 (1998).

[16] M. S. Daw and M. I. Baskes, Phys. Rev. Lett. 50, 1285 (1983).

[17] M. W. Finnis and J.E. Sinclair, Philos. Mag. A 50, 45 (1984).

[18] J. J. Palacios, A. J. Pérez-Jiménez, E. Louis, and J. A. Vergés, Phys. Rev. B 64, 115411 (2001); J. J. Palacios, A. J. Pérez-Jiménez, E. Louis, E. SanFabián, and J. A. Vergés, Phys. Rev. B 66, 035322 (2002); http://www. guirisystems.com/alacant. 